\title{
Identification of the Target Cells of Orientia tsutsugamushi in Human Cases of Scrub Typhus
}

\author{
Cecilia G. Moron, Vsevolod L. Popov, Hui-Min Feng, Douglas Wear, David H. Walker \\ Department of Pathology, University of Texas Medical Branch at Galveston, Galveston, Texas (CGM, VLP, \\ HMF, DHW); Instituto Nacional de Salud, Lima, Peru (CGM); and Armed Forces Institute of Pathology, \\ Washington, D.C. (DW)
}

\begin{abstract}
Orientia tsutsugamushi is the etiologic agent of scrub typhus, a chigger-borne zoonosis that is a highly prevalent, life-threatening illness of greatest public health importance in tropical Asia and the islands of the western Pacific Ocean. The target cell of this bacterium is poorly defined in humans. In this study, $O$. tsutsugamushi were identified by immunohistochemistry using a rabbit polyclonal antibody raised against $O$. tsutsugamushi Karp strain in paraffin-embedded archived autopsy tissues of three patients with clinical suspicion of scrub typhus who died during World War II and the Vietnam War. Rickettsiae were located in endothelial cells in all of the organs evaluated, namely heart, lung, brain, kidney, pancreas, and skin, and within cardiac muscle cells and in macrophages located in liver and spleen. Electron microscopy confirmed the location of rickettsiae in endothelium and cardiac myocytes.
\end{abstract}

KEY WORDS: Immunohistochemistry, Orientia tsutsugamushi, Rickettsia, Scrub typhus.

Mod Pathol 2001;14(8):752-759

Scrub typhus is a chigger-borne zoonosis that is of greatest public health importance in tropical Asia and the islands of the western Pacific Ocean. It became known to the West during World War II, when large numbers of cases occurred in soldiers of both sides in the Pacific and Asian theater. Outbreaks were associated with the early stage of development of a camp when chiggers were plentiful, with variable case-fatality rates ranging from $<1 \%$ to $>30 \%(1,2)$. Among indigenous populations, it is

Copyright (C) 2001 by The United States and Canadian Academy of Pathology, Inc.

VOL. 14, NO. 8, P. 752, 2001 Printed in the U.S.A.

Date of acceptance: February 27, 2001.

This work was supported by a Fogarty Grant for International Training and Research in Emerging Infectious Diseases (ITREID).

Address reprint requests to: David H. Walker, M.D., Department of Pathology, University of Texas Medical Branch, 301 University Blvd., Galveston, TX 77555-0609; e-mail: dwalker@utmb.edu; fax: 409-772-2500. a frequent cause of febrile illness leading to admission to a hospital (3). Orientia (formerly Rickettsia) tsutsugamushi, the etiologic agent of scrub typhus, is maintained in nature by highly efficient transovarial transmission in trombiculid mites and is transmitted to humans by the larval mite (chigger) during feeding. Although O. tsutsugamushi, a member of the family Rickettsiaceae, is phylogenetically related to the genus Rickettsia with 90.2 to $90.6 \%$ homology by $16 \mathrm{~S}$ rRNA gene sequences, its cell wall construction differs substantially and is distinguishable ultrastructurally (4-6). Although the cell wall of Rickettsia contains abundant, immunodominant lipopolysaccharides, outer membrane protein B, a 17-kDa lipoprotein, and, for spotted fever group rickettsiae, outer membrane protein $\mathrm{A}, O$. tsutsugamushi contains nothing even analogous to these components. The principal cell wall constituents of $O$. tsutsugamushi are the major strainvariable $56-\mathrm{kDa}$ protein as well as antigenically variable 110-, 47-, and 25-kDa proteins (7-11).

The pathogenesis of scrub typhus is even less well defined than that of the spotted fever and typhus group rickettsioses. Between 1916 and 1922, Wolbach and colleagues $(12,13)$ developed and applied modifications of the Giemsa stain to the demonstration of $R$. rickettsii and $R$. prowazekii, detecting intracellular rickettsiae in endothelial cells of cutaneous biopsies and necropsies of patients with Rocky Mountain spotted fever and epidemic louseborne typhus, respectively. However, the target cell or cells of Orientia tsutsugamushi are essentially undefined. In a study of 78 autopsies of cases of scrub typhus, Allen and Spitz (14) evaluated all the tissues of six appropriately fixed cases by Wolbach's modification of the Giemsa stain and detected rickettsiae in endothelial cells of only one site, an eschar, in a single case and in macrophages of a smear of spleen in a single case. Settle et al. (15) reported the demonstration of orientiae only in smears of human pericardial, pleural, and peritoneal fluid. It is difficult to understand the pathology and pathophysiology of a disease for which the 
locations of the infection in the organs are unknown, particularly for an obligately intracellular infectious agent. Thus, using immunohistochemistry (IHC) and electron microscopy, this investigation was undertaken to detect $O$. tsutsugamushi in formalin-fixed, paraffin-embedded autopsy tissues from three U.S. Army soldiers who died with clinical suspicion of scrub typhus during World War II and the Vietnam War, as well as in experimentally inoculated animals.

\section{CASE REPORTS}

\section{Case 1}

On November 7, 1943, generalized myalgia, malaise, headache, and enteritis developed in this 35year-old white male soldier serving in New Guinea. On the second day of illness, before presentation for medical attention, he noticed an eschar on the right upper arm that was $1 \mathrm{~cm}$ in diameter, with a black, necrotic ulceration and peripheral edema. He was admitted to the hospital, where physical examination revealed tender right axillary lymphadenopathy and an erythematous macular rash over the chest and abdomen. On Day 10 of illness, he showed disorientation, hyperreflexia, generalized lymphadenopathy, and a rising fever. On Day 13 , the patient became completely irrational, there was generalized hypertonicity of all muscle groups, early Cheyne-Stokes breathing, blood pressure of 105/62 mm Hg, pulse at 120 beats/minutes, and a soft systolic murmur over the mitral area. Later that day he developed ileus. The next day, he became increasingly dyspneic and tachycardic, developed a generalized clonic seizure, and died. Necropsy revealed vasculitis and perivasculitis in heart, brain, and kidneys; myocarditis; interstitial pneumonia with alveolar edema and hemorrhages; splenomegaly; and an eschar on the right upper arm. O. tsutsugamushi was isolated from postmortem cardiac blood by inoculation of mice.

\section{Case 2}

This 33-year-old soldier who had been working as a laborer on the roads in New Guinea was admitted to the hospital on February 24, 1945 complaining of having had malaise, anorexia, headache, and fever for 2 days. Physical examination revealed fever, a blood pressure of 94/60, and moderate conjunctival injection, and after admission, he developed a dry cough. On Day 9 of illness, rales were detected at the left base, the sputum showed pneumococci, the temperature was $103^{\circ} \mathrm{F}\left(39.4^{\circ} \mathrm{C}\right)$, and penicillin therapy was begun. On the 14th day of illness, the patient who had been lethargic seemed more alert, and his blood pressure was 120/60 mm Hg. Sud- denly, he complained of nervousness, suffered a short, generalized, convulsive seizure, and expired. Necropsy revealed vasculitis and perivasculitis in heart, brain, and kidneys; interstitial myocarditis; severe interstitial pneumonitis with hemorrhages; splenic hemorrhages; hepatic necrosis with portal triaditis and perivasculitis; interstitial nephritis with multiple hemorrhages; and an eschar.

\section{Case 3}

This 21-year-old soldier was admitted to a hospital in Vietnam on September 28, 1965 with a 3-day illness with a prodrome of weakness, anorexia, and headache followed by chills and fever for 48 hours. On physical examination, his temperature was $105^{\circ} \mathrm{F}\left(40.5^{\circ} \mathrm{C}\right)$ and blood pressure, $110 / 70 \mathrm{~mm} \mathrm{Hg}$. He was alert and cooperative, and diffuse inspiratory and expiratory wheezes were heard throughout both lung fields. The rest of the examination was normal. On Day 4 of illness, he was diagnosed with falciparum malaria by blood smear. His illness did not respond to pyrimethamine treatment. A maculopapular rash was observed on the 6th day of illness. Oral quinine was initiated on Day 5 of illness and was changed to intravenous treatment 3 days later. He developed a bleeding tendency with coffee-ground emesis, an hematocrit of 40 , white blood cells per cell count of 1000 cells per $\mu$, bleeding time of 5 minutes, and absence of platelets on peripheral blood smear. He suffered deafness, tachypnea, dyspnea, and hematemesis, passed large bloody stools, became incontinent and very restless, and died on the 12th day of illness. During his course, his body weight increased from 135 to 145 pounds. Necropsy revealed bleeding in the lungs, stomach, bowel, brain, and diaphragm; splenomegaly; and hepatomegaly with fatty metamorphosis.

\section{MATERIALS AND METHODS}

Autopsy specimens were archived as formalinfixed, paraffin-embedded tissue blocks at the Armed Forces Institute of Pathology in Washington, D.C. Paraffin sections $4 \mu \mathrm{m}$ in thickness were cut from blocks of heart, lung, spleen, liver, pancreas, kidney, and brain from Case 1; from lung, spleen, liver, kidney, and brain from Case 2; and from heart, lung, spleen, pancreas, kidney, brain, and skin from Case 3. Sections were also cut from blocks of heart, lung, liver, spleen, kidney, and testes from two Balb/c mice previously inoculated intraperitoneally with a tissue culture suspension of $O$. tsutsugamushi, one of them with Karp strain and the other with Gilliam strain. The mouse infected with Karp strain was sacrificed on Day 6 after inoculation, and the one infected with Gilliam strain, on 
Day 8. We used a polyclonal antibody raised against O. tsutsugamushi, Karp strain, developed in the Rickettsial and Ehrlichial Diseases Research Laboratory, University of Texas Medical Branch, as follows: a rabbit was injected intraperitoneally with $1.0 \mathrm{~mL}$ of purified O. tsutsugamushi. After 1 month, a booster immunization was administered, and 10 days later, serum was obtained for antibody titration. The IFA titer of the serum was 1:1280.

The sections were placed in poly-L-lysine, silanecoated slides and incubated at $70^{\circ} \mathrm{C}$ for 20 minutes, then rehydrated in water and digested with antigen retrieval solution (DAKO, Carpinteria, CA) for $20 \mathrm{~min}$ utes at $99^{\circ} \mathrm{C}$. The slides were incubated in $3 \%$ aqueous hydrogen peroxide for 30 minutes to quench endogenous peroxidase activity and rinsed in deionized water. Nonspecific binding of antibody was blocked by incubating specimens with normal goat serum and avidin blocking reagent (Vector Laboratories, Burlingame, CA) mixture (1:10) for 30 minutes. The antiOrientia antibody was placed on specimens at a dilution of 1:500 with DAKO diluent made up with biotin blocking reagent and incubated at room temperature for 30 minutes. This step was followed by successive 15-minute incubations with biotinylated anti-rabbit IgG (1:800) and the streptavidin reagent (DAKO, Carpinteria, CA). Color development was obtained by incubation for 5 minutes with diaminobenzidine (3,3'-diaminobenzidine). Sections were counterstained with hematoxylin, dehydrated, and mounted in Permount.

IHC controls consisted of the following: (1) substitution of normal rabbit serum for the primary antibody to rule out nonspecific binding; (2) substitution of anti-spotted fever group polyclonal antibody and monoclonal antibody to typhus group LPS for the primary antibody to rule out crossreactions; (3) evaluation of human tissue known not to contain rickettsiae; and (4) evaluation of tissues of mice known to be infected with Orientia as positive controls.

An area of myocardium of Patient 1 with the presence of O. tsutsugamushi identified in histochemically stained slides was marked on the paraf- fin block from which the section was made. A piece of tissue was cut out from the rickettsiae-containing area, deparaffinized in xylene, fixed in $1 \% \mathrm{O}_{\mathrm{S}} \mathrm{O}_{4}$ in 0.1 -M cacodylate buffer, stained en bloc in $2 \%$ aqueous uranyl acetate, dehydrated in ethanol, and embedded in PolyBed 812 (Polysciences, Warrington, PA). Ultrathin sections were cut with a Reichert Ultracut S ultramicrotome (Leica, Deerfield, IL), stained with lead citrate, and examined in a Philips 201 electron microscope (Philips Electron Optics, Eindhoven, The Netherlands) at $60 \mathrm{kV}$.

\section{RESULTS}

\section{Immunohistochemical Localization of O. tsutsugamushi}

The anti-Orientia immunohistochemical method demonstrated the bacteria in macrophages within the tissue sections from the mouse inoculated with Karp strain and also from that inoculated with Gilliam strain (Fig. 1). The rickettsiae were located within macrophages that had infiltrated the capsules of the liver and spleen as well as in splenic macrophages.

The basic histopathologic lesions in the human autopsy specimens were disseminated mononuclear perivasculitis, one of the most important lesions being interstitial pneumonia with hemorrhage and alveolar edema. The IHC staining of these specimens detected Orientia in endothelial cells of all of the organs evaluated, including in many blood vessels without perivascular mononuclear infiltrates (Fig. 2, A-E). O. tsutsugamushi was also detected within macrophages located in liver and spleen (Fig. 2, D, F). An unexpected finding was the identification of intracellular bacteria within well preserved cardiac muscle fibers in Cases 1 and 3 (Fig. 2E). The IHC assay showed no staining of Orientia when the primary antibody was replaced by normal rabbit serum or by anti-SFG or anti-typhus group rickettsial antibody. There was also no staining when the tissue

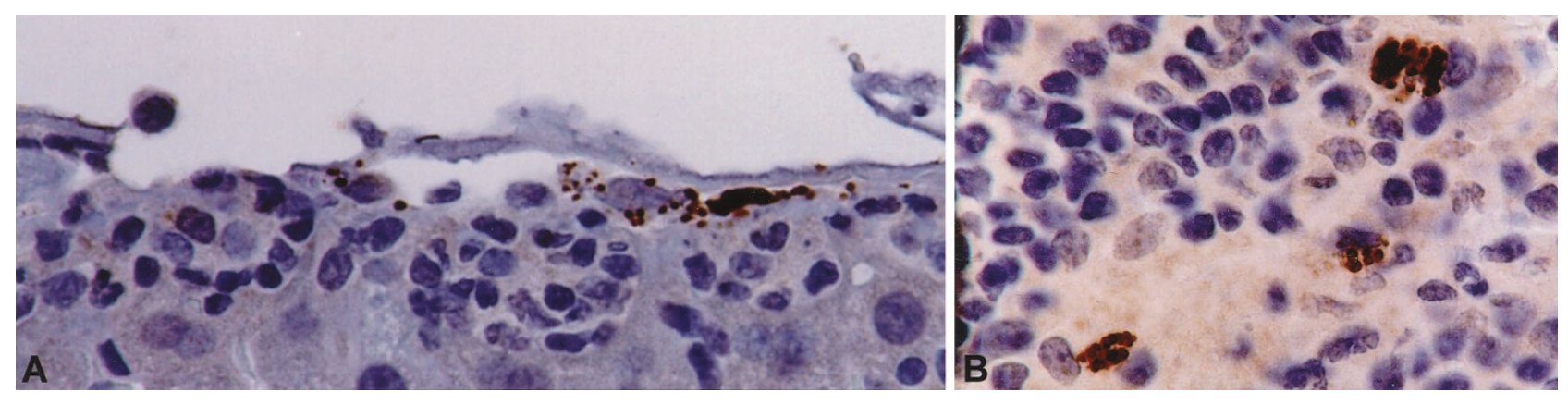

FIGURE 1. Immunohistochemically stained O. tsutsugamushi in macrophages in (A) the liver capsule (240×) and (B) the splenic parenchyma of an experimentally infected mouse $160 \times$. 

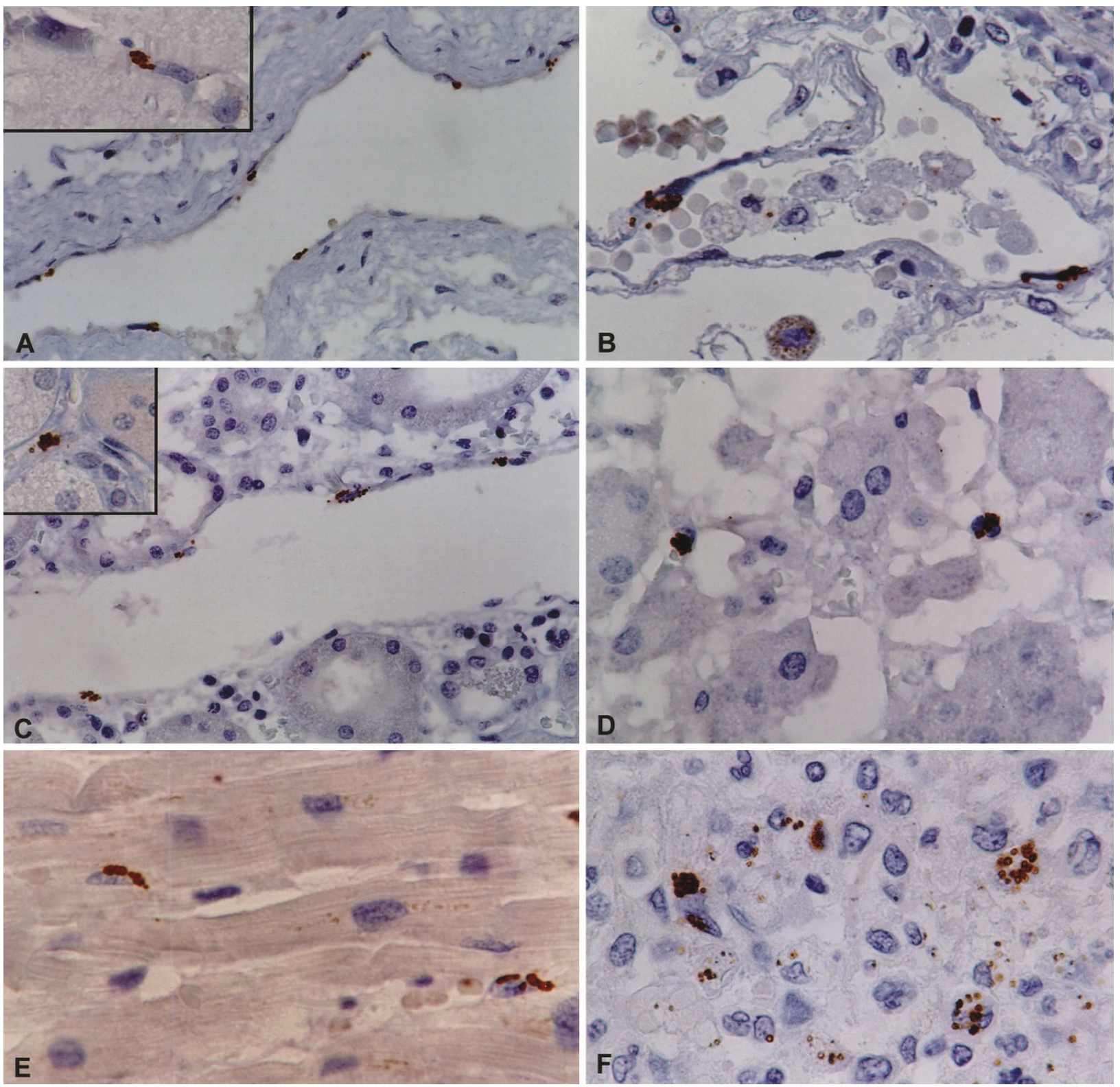

FIGURE 2. Immunohistochemically stained O. tsutsugamushi in endothelium in the brain of Case 2 (A [100×]; inset, $160 \times)$, in the lung of Case 3 (B $[120 \times])$, and kidney of Case $2(\mathbf{C}[100 \times])$ and Case $1(\mathbf{C}$ inset $[120 \times])$. Liver of Case 3 showing antigen of O. tsutsugamushi in Kupffer cells (D $[160 \times])$. Spleen of Case 3 showing $O$. tsutsugamushi in macrophages in the red pulp $(\mathbf{F}[160 \times])$. The heart of Case 3 showing location of $O$. tsutsugamushi in cardiac muscle cells and also in endothelium (E [160×]).

evaluated was human tissue not infected with $O$. tsutsugamushi.

\section{Ultrastructural Observations}

Orientia tsutsugamushi were found in endothelial cells of myocardial capillaries and in cardiac myocytes. Preservation of ultrastructural details in the sections was far from optimal as a result of formalin fixation and paraffin embedding, but Orientia were recognizable and identifiable inside the cells.

They were consistently found in the cytoplasm of capillary endothelial cells (Fig. 3A, arrows), which were observed to be heavily infected, containing several bacteria. A bacterium appeared to be in the process of exiting the host cell by budding (Fig. 3A, arrowhead). The budding rickettsia appeared to be surrounded by the plasma membrane of the host cell (Fig. 3, B-C, arrows). This mode of exit from the host cell is very characteristic of Orientia $(4,16)$. Another Orientia-specific feature was the thickness of the outer leaflet of the outer membrane of the cell wall (Fig. 3, C-D, arrowheads), which is thicker than the inner leaflet, contrary to what is observed in bacteria belonging to the genus Rickettsia. This ultrastructural feature is diagnostic for the genus Orientia (5). The rickettsial cytoplasmic membrane was generally poorly preserved in these samples but 

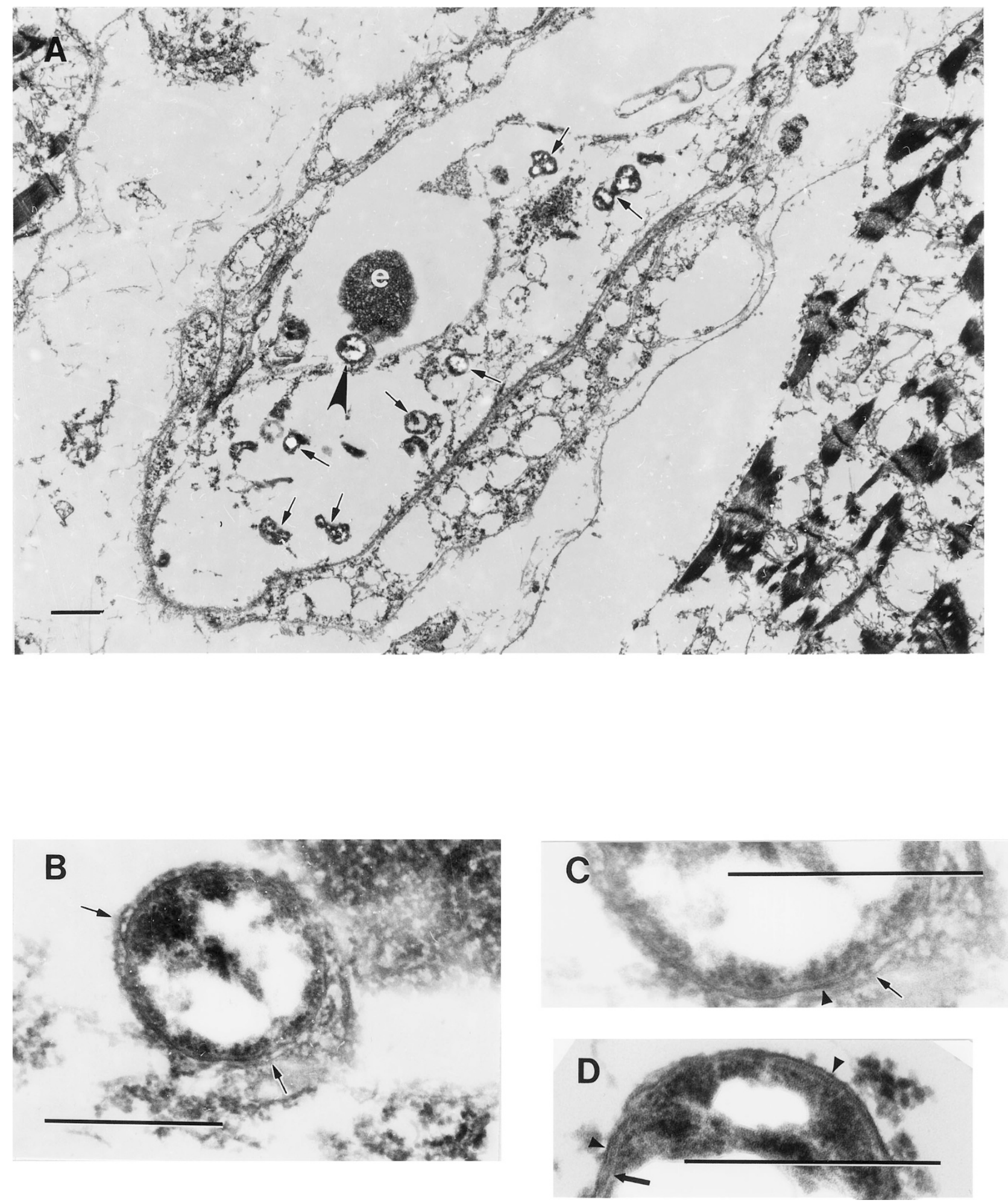

FIGURE 3. O. tsutsugamushi in an endothelial cell of a myocardial capillary. A, The capillary endothelial cell contains several bacteria in its cytoplasm (arrows). One bacterium appears to be budding from the endothelial cell surface (arrowhead). E-erythrocyte in the capillary lumen. Bar $=$ $1 \mu \mathrm{m}$. B, Higher magnification of a budding Orientia cell, surrounded by the plasma membrane (arrows) of the endothelial cell. Bar $=0.5 \mu \mathrm{m}$. C, Part of the cell wall of the rickettsia shown in Figure 3B demonstrating a thicker outer leaflet of the cell wall (arrowhead). Arrow indicates host cell membrane surrounding the bacterium. Bar $=1 \mu \mathrm{m}$. D, Part of an envelope of an orientia in another endothelial cell showing thicker outer leaflet of the outer membrane of the cell wall (arrowhead) and a cytoplasmic membrane $(($ arrow $)$. Bar $=1 \mu \mathrm{m}$.

could be identified in some organisms (Fig. 3D, arrow).

Bacteria with similar ultrastructure were also regularly found in the cytosol of cardiac myocytes, usually between the bundles of muscle filaments (Fig. 4, A-B, arrows). These organisms also had a typical thick outer leaflet of the outer membrane of the cell wall (Figure 4, C-D, arrowheads). They were 

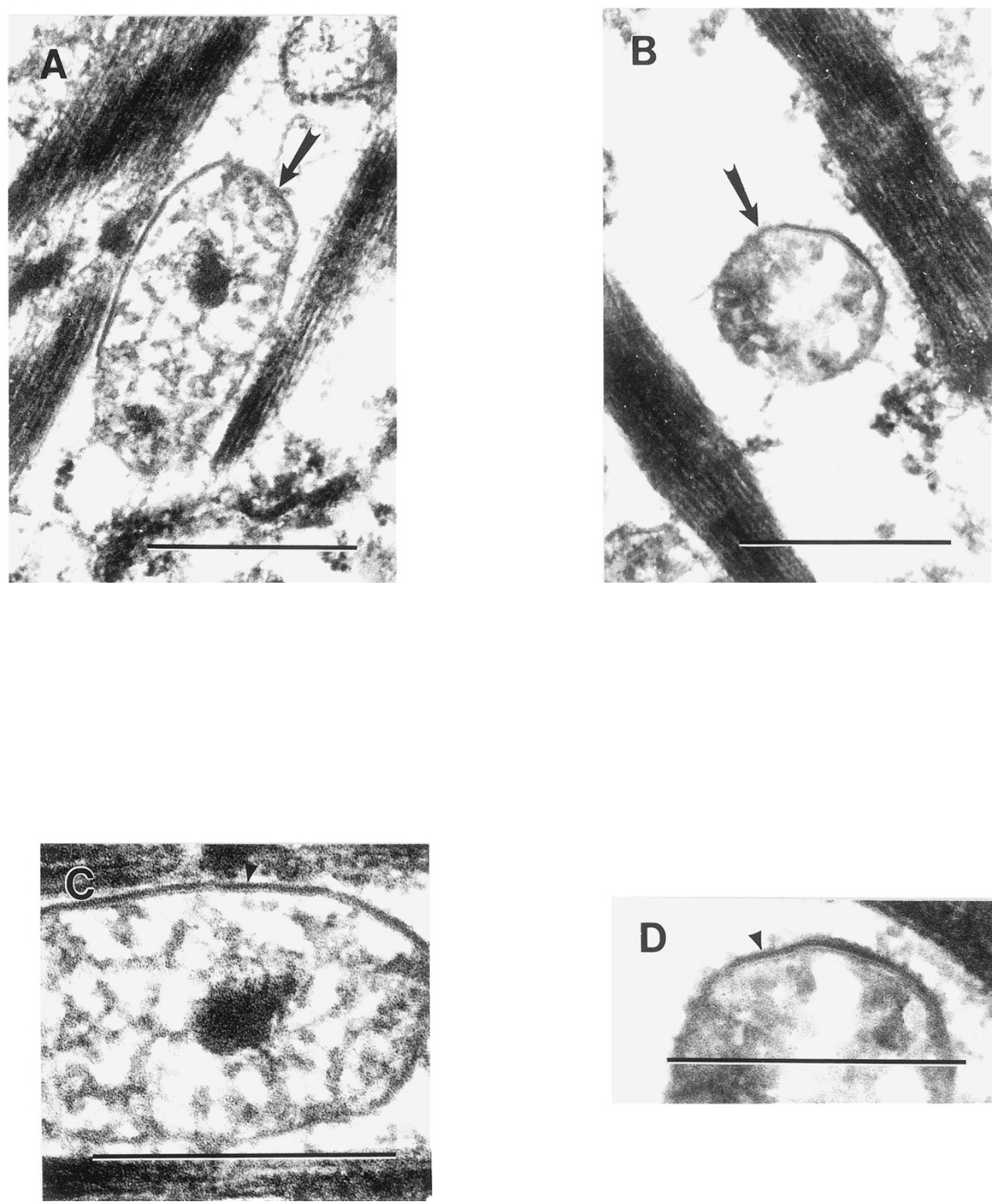

FIGURE 4. O. tsutsugamushi in the cardiac myocytes of patient 1 . Bar $=0.5 \mu \mathrm{m}$. A, B, Orientia cells (arrows) in the cytosol lying between the myofibrils of cardiac myocytes. C, D, Higher magnification of Orientia cells showing the typical thicker outer leaflet of the cell wall (arrowheads).

usually located as single rickettsiae rather than in groups as in endothelial cells, and they were clearly distinguishable from mitochondria.

These ultrastructural data confirm the observations from immunohistochemically stained sections that O. tsutsugamushi can infect endothelial cells and cardiac myocytes in patients with scrub typhus.

\section{DISCUSSION}

The discovery that $O$. tsutsugamushi infects principally endothelial cells in all of the organs available for examination with smaller quantities of infected macrophages and cardiac myocytes is a substantial addition to our foundation of knowledge of scrub typhus. The fundamental pathophysiology of Rocky Mountain spotted fever is that of increased vascular permeability, edema, hypovolemia, and hypoperfusion of some organs such as the kidneys (17). Although the pathogenesis of scrub typhus may be very complex and involve immune and inflammatory mediators such as cytokines, prostaglandins, leukotrienes, and kinins, the key factor of disseminated endothelial infection has now been established for tsutsugamushi disease as well as the rick- 
ettsioses. Indeed, Case 3 was apparently associated with generalized edema, and pleural, pericardial, and peritoneal effusions were reported in a substantial portion of fatal cases necropsied during World War II $(15,18)$. The histopathology of scrub typhus was investigated carefully in reports of 27 , 31,55 , and 78 cases $(14,15,18,19)$. The consensus in 1945 was that disseminated vasculitis with perivasculitis was the paramount lesion, with involvement of the brain and lungs being the most important factors in a fatal outcome. Vascular damage was manifested prominently by hemorrhagic phenomena, but thrombi were notably quite rare. At that time, the issue of myocardial damage and potentially acute and chronic cardiac insufficiency owing to the histologically evident interstitial inflammation was of particular concern. The identification of O. tsutsugamushi in cardiac myocytes reopens this question. In Levine's study, which focused on microscopic examination of the heart in 31 cases, six were perfectly normal, and 13 with inflammatory infiltrates showed intact heart muscle fibers (18). Twelve cases showed injury to cardiac myocytes, including several with frank focal necrosis that was rarely severe. No measurements of cardiac dynamics were available in that era to distinguish whether the basis of pulmonary edema and peripheral edema was congestive heart failure or noncardiogenic, owing to endothelial injurybased increased vascular permeability in the lungs. Levine concluded on the basis of autopsies of scrub typhus victims who died substantially later that the myocardial changes "eventually disappear, leaving no trace of previous damage" $(18$, p. 327). We did not observe necrosis of any cardiac myocytes that were infected with O. tsutsugamushi.

The demonstration of endothelial cells as the major target cells of O. tsutsugamushi in scrub typhus raises the issue of the appropriateness of animal model systems in which endothelial cells have not been reported to be infected. Mice infected with $O$. tsutsugamushi have been used extensively, particularly in studies of anti-Orientia immune mechanisms (20-26). Careful immunofluorescent studies of infected mice revealed that the route of inoculation determined the distribution of the organisms $(27,28)$. Intraperitoneal inoculation resulted in infection predominantly of the peritoneal lining and exudate. Intracerebral inoculation caused infection localized in the leptomeninges. In contrast, intramuscular or subcutaneous inoculation produced disseminated infection involving "Kupffer cells, macrophages, and other forms of mesothelial connective tissue" in the liver, spleen, lungs, kidneys, lymph nodes, thymus, and adrenal glands (27, p. 777). Even considering the limitations of determining the identity of cell types by fluorescence microscopy, one must conclude that there are serious reservations regarding the appropriateness of these mouse models in elucidating the mechanisms of immunity or pathogenesis of human infection with O. tsutsugamushi.

Similar questions pertain to cell culture models of O. tsutsugamushi infection. Geng and Jerrells (23) demonstrated that recombinant tumor necrosis factor- $\alpha$ inhibited intracellular growth of $O$. tsutsugamushi (Karp strain) in a mouse embryo cell line and in macrophages derived from peritoneal exudate or bone marrow. However, gamma interferon inhibited the growth of $O$. tsutsugamushi only in the murine macrophages and not in the mouse embryo cell line. The cell type was critical in the outcome, in other words, in growth or inhibition of the organisms. Our present study emphasizes that understanding intracellular anti-Orientia mechanisms must require investigations in endothelial cells as well as macrophages, including studies in human cells.

Previous studies in our laboratory have demonstrated that IHC is an effective method for detecting $R$. prowazekii, $R$. typhi, $R$. conorii, $R$. akari, and $R$. rickettsii in formalin-fixed paraffin-embedded tissues (29-32). In the present study, the reliable demonstration of $O$. tsutsugamushi by IHC with an antiOrientia antibody confirmed this method as an improved diagnostic tool, as shown by the detection of these organisms in 35- to 57-year-old samples.

It is remarkable that the rabbit polyclonal antibody detected both Karp and Gilliam strains in infected mice as well as the unknown strains of our three human cases. An important antigen for the detection of the spotted fever and typhus groups of Rickettsia is the respective group-specific lipopolysaccharides. The absence of lipopolysaccharide in $O$. tsutsugamushi and the antigenic variation of the $56-\mathrm{kDa}$ major surface protein make the detection of this organism a special challenge $(8,33)$. Indeed it is possible that this particular polyclonal antibody to O. tsutsugamushi might not detect all of the multitude of genetically divergent strains. However, within a particular geographic locus where a limited number of strains are in circulation, antibodies could be produced for diagnostic use in identification of O. tsutsugamushi in cutaneous biopsies of rash or eschar. Because only a fraction of patients manifest these lesions, immunohistochemical diagnosis cannot be achieved in all cases by this approach. However, in critically ill patients who are admitted late in the course of illness, it would be highly useful to establish the precise diagnosis in as timely a manner as possible to ensure that appropriate treatment is administered, inappropriate treatment is avoided, and attention is alerted for the well-established manifestations and complications of scrub typhus. Evaluation of IHC for diag- 
nostic identification of $O$. tsutsugamushi would be particularly important in areas of the world where scrub typhus is endemic.

Acknowledgments: We thank Mr. Brent Norris and Ms. Barbara Hightower for their technologic contributions to the IHC staining, Ms. Violet Han for expert assistance in electron microscopy, Ms. Kelly Cassity for expert secretarial assistance in the preparation of this article, and Mr. Thomas Bednarek for assistance in preparation of illustrations.

\section{REFERENCES}

1. Watt G, Walker DH. Scrub typhus. In: Guerrant RL, Walker DH, Weller PF, editors. Tropical infectious diseases: principles, pathogens, and practice. Vol 1. Philadelphia: Churchill Livingstone; 1999. p. 592-7.

2. Brown GW. Scrub typhus: Pathogenesis and clinical syndrome. In: Walker DH, editor. Biology of rickettsial diseases. Vol I. Boca Raton, FL: CRS Press; 1988. p. 93-100.

3. Brown GW, Robinson DM, Huxsoll DL, Ng TS, Lim KJ. Scrub typhus: a common cause of illness in indigenous populations. Trans R Soc Trop Med Hyg 1977;70:444-8.

4. Tamura A, Ohashi N, Urakami H, Miyamura S. Classification of Rickettsia tsutsugamushi in a new genus, Orientia gen. nov., as Orientia tsutsugamushi comb. nov. Int J Syst Bacteriol 1995;45:589-91.

5. Silverman DJ, Wisseman CL Jr. Comparative ultrastructural study on the cell envelopes of Rickettsia prowazekii, Rickettsia rickettsii, and Rickettsia tsutsugamushi. Infect Immun 1978;21:1020-3.

6. Ohashi N, Fukuhara M, Shimada M, Tamura A. Phylogenetic position of Rickettsia tsutsugamushi and the relationship among its antigenic variants by analyses of 16S rRNA gene sequences. FEMS Microbiol Lett 1995;125:299-304.

7. Tamura A, Ohashi N, Urakami H, Takahashi K, Oyanagi M. Analysis of polypeptide composition and antigenic components of Rickettsia tsutsugamushi by polyacrylamide gel electrophoresis and immunoblotting. Infect Immun 1985;48:671-5.

8. Ohashi N, Nashimoto H, Ikeda H, Tamura Al. Diversity of immunodominant 56-kDa type-specific antigen (TSA) of Rickettsia tsutsugamushi. Sequence and comparative analyses of the genes encoding TSA homologues from four antigenic variants. J Biol Chem 1992;267:12728-35.

9. Oaks EV, Stover CK, Rice RM. Molecular cloning and expression of Rickettsia tsutsugamushi genes for two major protein antigens in Escherichia coli. Infect Immun 1987;55:1156-62.

10. Oaks EV, Rice RM, Kelly DJ, Stover CK. Antigenic and genetic relatedness of eight Rickettsia tsutsugamushi antigens. Infect Immun 1989;57:3116-22.

11. Hanson B. Identification and partial characterization of Rickettsia tsutsugamushi major protein immunogens. Infect Immun 1985;50:603-9.

12. Wolbach SB, Todd JL, Palfrey FW. Pathology of typhus in man. The etiology and pathology of typhus. Cambridge, MA: Harvard University Press; 1922. p. 152-221.

13. Wolbach SB. Studies on Rocky Mountain spotted fever. J Med Res 1919;41:1-70.

14. Allen AC, Spitz S. A comparative study of the pathology of scrub typhus (tsutsugamushi disease) and other rickettsial diseases. Am J Pathol 1945;21:603-61.

15. Settle EB, Pinkerton H, Corbett AJ. A pathologic study of Tsutsugamushi disease (Scrub typhus) with notes on clinicopathologic correlation. J Lab Clin Med 1945;30:639-61.
16. Ewing EP Jr, Takeuchi A, Shiral A, Osterman JV. Experimental infection of mouse peritoneal mesothelium with scrub typhus rickettsiae: an ultrastructural study. Infect Immun 1978;19:1068-75.

17. Harrell GT, Aikawa JK. Pathogenesis of circulatory failure in Rocky Mountain spotted fever. Alteration in the blood volume and the thiocyanate space at various stages of the disease. Arch Intern Med 1949;83:331-47.

18. Levine HD. Pathologic study of thirty-one cases of scrub typhus fever with especial reference to the cardiovascular system. Am Heart J 1946;31:314-28.

19. Park JH, Hart MS. The pathology of scrub typhus. Am J Clin Pathol 1946;16:139-49.

20. Jerrells TR. Mechanisms of immunity of Rickettsia species and Coxiella burnetii. In: Walker DH, editor. Biology of rickettsial diseases. Boca Raton, FL: CRC Press; 1988. p. $79-100$.

21. Shirai A, Catanzaro PJ, Phillips SM, Osterman JV. Host defenses in experimental scrub typhus: role of cellular immunity in heterologous protection. Infect Immun 1976;14:3946.

22. Jerrells TR, Osterman JV. Role of macrophages in innate and acquired host resistance to experimental scrub typhus infection of inbred mice. Infect Immun 1982;37:1066-73.

23. Geng PQ, Jerrells TR. The role of tumor necrosis factor in host defense against scrub typhus rickettsiae. I. Inhibition of growth of Rickettsia tsutsugamushi, Karp strain, in cultured murine embryonic cells and macrophages by recombinant tumor necrosis factor-alpha. Microbiol Immunol 1994;538: 703-11.

24. Jerrells TR, Geng PQ. The role of tumor necrosis factor in host defense against scrub typhus rickettsiae. II. Differential induction of tumor necrosis factor-alpha production by Rickettsia tsutsugamushi and Rickettsia conorii. Microbiol Immunol 1994;38:713-9.

25. Rollwagen FM, Dasch GA, Jerrells TR. Mechanisms of immunity to rickettsial infection: characterization of a cytotoxic effector cell. J Immunol 1986;136:1418-21.

26. Catanzaro PJ, Shirai A, Hilderbrandt PK, Osterman JV. Host defenses in experimental scrub typhus: histopathological correlates. Infect Immun 1976;13:861-75.

27. Kundin WD, Liu C, Harmon P, Rodina P. Pathogenesis of scrub typhus infection (Rickettsia tsutsugamushi) as studied by immunofluorescence. J Immunol 1964;93:772-81.

28. Murata M, Sudo K, Suzuki K, Aoyama Y, Nogami S, Tanaka $\mathrm{H}$, et al. Proliferating sites of Rickettsia tsutsugamushi in mice by different routes of inoculation evidenced with immunofluorescence. Jpn J Exp Med 1985;55: 193-9.

29. Walker DH, Parks FM, Betz TG, Taylor JP, Muehlberger JM. Histopathology and immunohistologic demonstration of the distribution of Rickettsia typhi in fatal murine typhus. Am J Clin Pathol 1989;91:720-4.

30. Walker DH, Feng HM, Ladner S, Billings AN, Zaki SR, Wear DJ, et al. Immunohistochemical diagnosis of typhus rickettsioses using an anti-lipopolysaccharide monoclonal antibody. Mod Pathol 1997;10:1038-42.

31. Walker DH, Hudnall SD, Szniawski WK, Feng H-M. Monoclonal antibody-based immunohistochemical diagnosis of rickettsialpox: the macrophage is the principal target. Mod Pathol 1999;12:529-34.

32. Walker DH, Popov VL, Wen J, Feng H-M. Rickettsia conorii infection of $\mathrm{C} 3 \mathrm{H} / \mathrm{HeN}$ mice: a model of endothelial-target rickettsiosis. Lab Invest 1994;70:358-68.

33. Amano K, Tamura A, Ohashi N, Urakami H, Kaya S, Fukushi K. Deficiency of peptidoglycan and lipopolysaccharide components in Rickettsia tsutsugamushi. Infect Immun 1987;55: $2290-2$. 